\title{
Implicações e benefícios da Smart Rehabilitation nas disfunções neurológicas: uma
}

\section{revisão narrativa}

\author{
Effects and benefits of Smart Rehabilitation in neurological dysfunction: a narrative review \\ Implicaciones y beneficios de la Rehabilitación Inteligente en disfunciones neurológicas: una \\ revisión narrativa
}

Recebido: 16/12/2021 | Revisado: 24/12/2021 | Aceito: 31/12/2021 | Publicado: 08/01/2022

\author{
Geraldo Carvalho Magalhães \\ ORCID: https://orcid.org/0000-0002-7367-3025 \\ Universidade Federal da Paraíba, Brasil \\ E-mail: geraldo_magalhaes2000@yahoo.com.br \\ Bárbarah Carolyne Moreira Rodrigues Antas \\ ORCID: https://orcid.org/0000-0002-7491-1830 \\ Universidade Federal da Paraíba, Brasil \\ E-mail: barbarahrodriigues@hotmail.com \\ Renata de Lima Martins \\ ORCID: https://orcid.org/0000-0003-4927-8153 \\ Universidade Federal da Paraíba, Brasil \\ E-mail: renatamarttins@ outlook.com \\ Camila Fernandes Pontes dos Santos \\ ORCID: https://orcid.org/0000-0002-9280-5628 \\ Universidade Federal da Paraíba, Brasil \\ E-mail: pontescamila6@gmail.com \\ Patrick Kervin de Almeida Chaves \\ ORCID: https://orcid.org/0000-0003-3823-4745 \\ Universidade Federal da Paraíba, Brasil \\ E-mail: patricker007@gmail.com \\ Adriana Costa-Ribeiro \\ ORCID: https://orcid.org/0000-0002-8940-9069 \\ Universidade Federal da Paraíba, Brasil \\ E-mail: accrc@academico.ufpb.br
}

\begin{abstract}
Resumo
Objetivo: Em todo o mundo a fisioterapia neurofuncional avança no propósito de prover novas ferramentas para auxiliar o processo de reabilitação de pacientes com disfunções e incapacidades neuromusculoesqueléticas. Nos dias de hoje, o processo de recuperação é potencializado pela implementação de dispositivos robóticos, eletrônicos e vestíveis (DREV). Essa revisão narrativa aborda os dispositivos inteligentes, discutindo o uso dos mesmos na população, categorizando-os e explorando-os quanto à aplicabilidade na fisioterapia dos DREV. Métodos: Artigos na língua inglesa e portuguesa da base de dados Pubmed, Scielo, Bireme, ResearchGate e outras fontes publicadas nos últimos 10 anos foram compilados e posteriormente comparados e analisados para determinar sua relevância e finalmente incluídos no artigo após revisão criteriosa. Resultados: Um total de 53 artigos sobre dispositivos inteligentes foram qualitativamente analisados e as informações sintetizadas. Essa revisão narrativa mostra que os DREV vêm ganhando mais espaço no processo de reabilitação fisioterapêutica e que podem ser utilizados em diversos segmentos do corpo humano por transformar a qualidade de vida dos usuários. Conclusão: Apesar das limitações e da disponibilidade dessa tecnologia à população mais pobre, acredita-se que os dispositivos são opções promissoras no campo da saúde. A implementação no espectro clínico hospitalar e acessibilidade dos DREV precisa de discussão interdisciplinar entre as várias categorias da saúde para que se tenha uma melhor oferta de cuidados às pessoas com condições neuromusculoesqueléticas.
\end{abstract}

Palavras-chave: Equipamentos de autoajuda; Fisioterapia; Distúrbios neurológicos.

\begin{abstract}
Aim: Worldwide, neurofunctional physiotherapy has advanced in the purpose of providing new devices in patient's rehabilitation process with neuro-musculoskeletal disorders and disabilities. Nowadays, rehabilitation is enhanced by the implementation of robotics, electronics and wearable devices which leads physical therapy treatment to a new level. This narrative review addresses smart devices subject by discussing its application in benefited population, categorizing them and exploring its applicability in physiotherapy. Methods: English and portuguese articles from database Pubmed, Scielo, Bireme, ResearchGate and others sources published in the last 10 years were compiled and posteriorly compared and analyzed to determine its relevance and finally included after criterious revision. Results: A
\end{abstract}


total of 53 articles about smart devices were qualitatively analyzed and the information synthesized. This narrative revision shows that this technology is gaining space on physical therapy rehabilitation process, besides it can be used in many parts of the human body, as well as of the changes in user's quality of living. Conclusion: Despite of its limitations and the lack of availability in poorest people, we believe that these devices are a promising options in the field of health care. Its implementation in clinical-hospital spectrum and availability demands interdisciplinary discussion among different health categories for a better delivery of care for those with neuro-musculoskeletal conditions.

Keywords: Self-help devices; Physiotherapy; Nervous system disease.

\section{Resumen}

Objetivo: La fisioterapia neurofuncional, en todo el mundo, avanza con el propósito de proporcionar nuevas herramientas que auxilien el proceso de rehabilitación de pacientes con disfunciones e incapacidades neuromusculoesqueléticas. Actualmente, el proceso de recuperación se potencia con la implantación de dispositivos robóticos, electrónicos y portátiles (DREV). Esta revisión bibliográfica aborda los dispositivos inteligentes y discute el uso de los mismos en la población, categorizándolos y explotando su aplicabilidad en la fisioterapia de los DREV. Métodos: Se recopilaron artículos en lengua inglesa y portuguesa de la base de datos Pubmed, Scielo, Bireme, ResearchGate y otras fuentes publicadas en los últimos 10 años y posteriormente se compararon y se analizaron para determinar su relevancia para finalmente incluirlos en el artículo después de una rigurosa revisión. Resultados: Se analizó un total de 53 artículos sobre dispositivos inteligentes cualitativamente y también se sintetizaron las informaciones. Esta revisión bibliográfica muestra que los DREV han ganado más espacio en el proceso de rehabilitación fisioterapéutica y que pueden utilizarse en diferentes segmentos del cuerpo humano ya que transforman la calidad de vida de los usuarios. Conclusión: A pesar de las limitaciones y de la disponibilidad de esta tecnología para la población más pobre, se cree que los dispositivos son opciones promisoras en el campo da salud. La implantación en el espectro clínico hospitalar y accesibilidad de los DREV necesitan una discusión interdisciplinar entre las diversas categorías de la salud para tener una oferta mejor de cuidados a las personas con condiciones neuromusculoesqueléticas.

Palabras-clave: Dispositivos de autoayuda; Fisioterapia; Enfermidade del sistema nervioso.

\section{Introdução}

Na última década, o raciocínio clínico em fisioterapia tem estado voltado para o desenvolvimento de sistemas para os fisioterapeutas tomarem decisões clínicas de forma rápida, eficaz e eficiente, em resposta às necessidades cada vez mais complexas das unidades de saúde e reabilitação. Na literatura, uma ampla gama de sistemas robóticos e tecnologias vestíveis foram introduzidas para ajudar as pessoas com condições neuromusculoesqueléticas. Para estabelecer uma discussão eficiente sobre as tecnologias existentes e como elas podem ser adaptadas para ajudar na atual situação de pandemia, é vantajoso discutir uma série de definições e maneiras de classificar tais tecnologias. Recentemente, os sistemas assistidos por tecnologia apresentam grande potencial para apoiar a reabilitação. Os sistemas de reabilitação inteligente baseados na Internet das Coisas (IOT) podem fornecer uma plataforma eficaz para interconectar vários recursos e alcançar a comunicação imediata. Sistema de monitoramento de saúde móvel baseado em computação em nuvem pode suportar diagnóstico descentralizado, compartilhamento de dados de saúde e interação entre os sistemas de maneira produtiva (Atashzar, 2021).

Uma sociedade mais permeável à diversidade questiona seus mecanismos de segregação e vislumbra novos caminhos de inclusão social para as pessoas com limitações funcionais. Este fato tem estimulado e fomentado novas pesquisas, inclusive com a apropriação dos acelerados avanços tecnológicos disponíveis na atualidade. Segundo essa perspectiva, conceitos como Tecnologia Assistiva (TA) surgem e ganham espaço no meio científico, principalmente na área da saúde, sob uma perspectiva multidisciplinar, descentralizada e heterogênea. Apesar de possuir outras atribuições, o campo da TA contribuiu para melhorar as habilidades funcionais de pessoas acometidas por danos leves ou graves e, dessa forma, proporciona mais autonomia e independência funcional. A área da TA surgiu no Brasil em novembro de 2006, quando o Comitê de Ajudas Técnicas (CAT) a propôs por meio da Portaria ${ }^{\circ} 142$. Em virtude da recente institucionalização da área representada pela TA, naturalmente há uma produção acadêmico-científica especializada e desassociada tanto em relação a aspectos de Investigação e Desenvolvimento (I\&D) quanto de Pesquisa e Desenvolvimento (P\&D) devido à pulverizada produção nos canais científicos (Brasil, 2009). 
A população que se beneficia da SR é formada por pessoas que apresentam algum tipo de disfunção do sistema nervoso central e/ou periférico, como também pode ser utilizada no tratamento de distúrbios musculoesqueléticos. O presente estudo abordará a utilização da SR nas disfunções do sistema nervoso e dos dispositivos que podem auxiliar no tratamento dessas doenças e que podem ser utilizadas por essa população.

A Paralisia Cerebral (PC) é uma doença crônica não progressiva, decorrente de lesões ou anormalidades no cérebro imaturo que cursa com distúrbios de movimento, postura e função motora. Desta forma, o comprometimento motor caracteriza-se como a principal manifestação na PC, ocasionando diferentes alterações na biomecânica corporal que afetam o desempenho funcional de várias maneiras. Diferentes abordagens terapêuticas podem ser utilizadas a fim de promover melhorias funcionais nessa população. Nesse contexto está inserida a TA como adjuvante a terapia convencional (Pasini et al., 2012).

No Acidente Vascular Cerebral (AVC), a função da extremidade superior distal é fortemente comprometida, de forma que dificulta a realização de atividades que outrora eram relativamente simples e corriqueiras, como por exemplo, as atividades de vida diária (AVDs). Dessa forma, funções básicas como segurar objetos, girar uma maçaneta ou chave em uma fechadura, usar telefone ou computador e escrever, se tornam difíceis de serem executadas. Wolf (2006 conforme citado em Shin $e t$ al., 2016) afirma que a extremidade superior distal é a última parte do corpo a se recuperar no AVC, sendo considerado como importante objetivo na reabilitação dessa população. Nesse contexto, a fisioterapia é inserida com vistas a maximizar os ganhos funcionais e melhorar a qualidade de vida das pessoas acometidas por AVC. Além do treinamento motor convencional, a inserção de novas tecnologias vem sendo implementadas, dentre elas está a realidade virtual (Shin et al., 2016).

As pessoas com Doença de Parkinson (DP) apresentam comprometimentos motores que progridem ao longo do tempo. A terapia medicamentosa é a mais utilizada para essa população, no entanto, por ser um tratamento com efeitos colaterais conhecidos, faz-se necessário a implementação de novas abordagens terapêuticas que minimizem os riscos e promovam mais funcionalidade aos indivíduos com DP (Mohammadi-Abdar et al., 2016).

O Traumatismo Crânio-encefálico (TCE) é considerado uma agressão ao cérebro, causado por uma força física externa, que pode produzir um estado diminuído ou alterado do nível de consciência e acarretar a comprometimentos das habilidades cognitivas e funcionais (Morgado \& Rossi, 2011) decorrentes de lesões anatômicas ou comprometimentos funcionais. Nesse contexto, a aplicação de TA que busque proporcionar melhora cognitiva, ganho de força e coordenação motora e, consequentemente, ganho de funcionalidade em pessoas que sofreram TCE faz-se relevante (Mendes et al., 2018).

Indivíduos com Esclerose Lateral Amiotrófica (ELA) apresentam modificações nas capacidades motoras, de cognição e comportamentais, causando complicações nas competências funcionais e gerando limitações de interação social (Leite et al., 2021). A perda da capacidade da fala é um obstáculo que surge com a progressão dessa doença. Desse modo, a comunicação alternativa ampliada é um meio para atender a necessidade desses indivíduos por intermédio de simbologias, grafias e frases pré-formadas e outros meios de comunicação que não são de modo tradicional, como a fala e os gestos. Para aplicação dessa alternativa pode-se utilizar uma tecnologia de baixo custo como pranchas de comunicação, ou de um custo alto como softwares próprios em computadores, tablets e aplicativos. Esse uso tem a finalidade da interação dos indivíduos no contexto social e familiar, promovendo melhor qualidade de vida e autoestima (Coimbra et al., 2018).

Na Lesão Medular (LM), ocorre o comprometimento da função motora, especialmente da capacidade de locomoção, a depender do nível e do tipo da lesão. Desta forma, essa condição pode ser devastadora com grande impacto na vida de uma pessoa. A literatura aponta que a fisioterapia convencional promove neuroplasticidade, melhorando a função motora dos indivíduos com LM. No entanto, o treinamento manual pode ser exaustivo e extenuante para os terapeutas. Por isso, estratégias utilizando dispositivos eletromecânicos automatizados podem ser introduzidos, com vistas a facilitar o processo de reabilitação 
em diversas maneiras que vão desde a melhora funcional do indivíduo até a redução da quantidade de pessoas envolvidas em um treino de marcha, por exemplo, que pode demandar até três terapeutas (Nam et al., 2017).

A Fisioterapia Neurofuncional atua no cuidado e no tratamento de disfunções do sistema nervoso central, e, constituise como área de atuação, na qual muito conhecimento, dedicação e trabalho são necessários para se garantir uma boa evolução dos casos problema que se apresentam. A depender do processo de plasticidade neuronal possibilitado com as intervenções fisioterapêuticas, os resultados podem ser impressionantes. Nesse contexto, faz-se mister, a implementação de dispositivos de TA como forma de incremento da capacidade funcional, autonomia e qualidade de vida dos pacientes. Os dispositivos de TA são de grande valia, aumentando a funcionalidade, autonomia e a qualidade de vida de pacientes com patologias neurológicas. Em contrapartida a esse avanço, ainda é possível observar a dificuldade em encontrar pesquisas científicas claras e bem definidas na literatura nacional acerca dessa temática. Portanto, o objetivo desse estudo é revisar a literatura científica no tocante ao uso da TA na reabilitação de pessoas com disfunção neurológica. Baseado no exposto, a seguinte questão norteadora se impõe: de que forma a tecnologia assistiva pode facilitar a avaliação, assistência e reabilitação de indivíduos com acometimentos neurológicos?

\section{Metodologia}

O presente estudo se trata de uma revisão da literatura narrativa, construída por meio de uma pesquisa bibliográfica descritiva, retrospectiva e qualitativa, embasada em publicações disponíveis nas bases de dados Pubmed (Public/Publisher MEDLINE), Scielo (Scientific Electronic Library Online), Bireme (Biblioteca Regional de Medicina) e ResearchGate. A coleta de dados levou em consideração o objeto de estudo, a partir da combinação dos descritores "Smart devices, "Smart rehabilitation" e "Walking", usando o operador boleano "and". Para uma busca mais específica sobre o tema, uma pesquisa manual com base nas referências de outros artigos também foi realizada.

Segundo Casarin et al. (2020) a Revisão Narrativa (RN) é uma forma não sistematizada de revisar a literatura. É importante para buscar atualizações a respeito de um determinado assunto dando ao revisor suporte teórico em curto período. Também pode ser útil na descrição do estado da arte de um assunto específico, sob o ponto de vista teórico ou contextual. Como a RN inclui um processo mais simplificado de revisar a literatura, a questão de pesquisa pode ser mais ampla ou pouco específica e abordar um tema de forma livre, sem rigor metodológico e por isso está sujeita aos vieses. Na RN não há obrigatoriedade de que os autores informem com detalhes os procedimentos ou critérios usados para selecionar e avaliar as referências incluídas na análise, pois a forma de seleção é variável e arbitrária (Casarin et al., 2020).

A pesquisa incluiu artigos científicos escritos em inglês ou português, publicados no período de 2011 até o ano de 2021 em revistas online que abordassem o tema Smart Rehabilitation, categorizando as tecnologias disponíveis e discutindo sua aplicabilidade. A partir da leitura dos resumos e dos artigos completos, foram encontrados 54 artigos, que se demonstraram úteis para contextualizar e/ou corroborar a utilização da Smart Rehabilitation. A coleta de dados aconteceu entre os meses de setembro e novembro de 2021.

\section{Resultados e Discussão}

\subsection{Categorias de Smart Rehabilitation}

A TA é uma área que abrange recursos e serviços para proporcionar ou ampliar habilidades funcionais, promovendo maior independência e inclusão. As áreas de recursos e serviços estão bem delimitadas na literatura, em que os recursos incluem desde órteses e próteses, a softwares e hardwares especiais para promover a acessibilidade. Os serviços envolvem profissionais que auxiliam na adaptação das pessoas aos recursos de TA. Dentre os vários profissionais envolvidos nos 
serviços, o fisioterapeuta tem papel importante, visto que lida diretamente com o movimento humano (Silva, Ledycnarf \& Morya, 2017).

Um dos caminhos utilizados pela TA são os dispositivos que colaboram com a mobilidade, como por exemplo, as cadeiras de banho, cadeiras de rodas, bengalas e andadores, que geralmente são recomendados para indivíduos com dificuldades na deambulação, como nos casos de AVC (Caro et al., 2018).

Há várias modalidades da robótica que podem ser usadas nas principais modalidades do espectro de cuidados de saúde (reabilitação, avaliação e assistência) necessários para pacientes com deficiências neuromusculoesqueléticas. As categorias podem ser definidas de acordo com a estrutura mecânica ou a modalidade de interação humano-robô.

Com o avanço tecnológico e o desenvolvimento de sensores sem fio com um baixo consumo de baterias, é viável uma ampla produção de dispositivos aceitáveis em variadas áreas, tal como, colaborando nos serviços de saúde que procuram dispositivos vestíveis não invasivos permitindo uma assistência ao paciente. Essa particularidade tecnológica ajuda especialmente os médicos, cuidadores e familiares na monitorização dos idosos, por serem um público-alvo de dispositivos vestíveis voltados para a saúde, possibilitando por meio dos sensores informações como sinais vitais e fisiológicos, atividades diárias, dados médicos e comunicação de emergência, isto é, com o intuito de promover um diagnóstico precoce do paciente, evitando imprevistos de emergência (Barreto et al., 2019).

Uma das categorias são as interfaces cérebro-máquina que permitem o reconhecimento da atividade neural no cérebro dos utilizadores para oferecer aos indivíduos com mobilidade limitada, devido um acidente ou por uma doença neurodegenerativa, um meio de determinar uma relação direta entre o cérebro e o dispositivo. Sendo assim, as interfaces cérebro-máquina embasadas em técnicas não invasivas, como o eletroencefalograma, têm possibilitado a esses indivíduos novas viabilidades em recuperar o controle das atividades de vida diária que de outro modo não realizariam, especialmente no campo da comunicação e controle do ambiente (Llorente, 2019).

\subsection{Dispositivos}

A Smart Rapael Glove TM (Neofect, Yong-in, Coreia) é um sistema de biofeedback projetado para a reabilitação da mão em pessoas com AVC. É composto por um dispositivo sensor em forma de luva e um aplicativo de software. O dispositivo sensor rastreia o movimento e o posicionamento da mão do usuário e reconhece os movimentos funcionais, como pronação/supinação do antebraço, flexão/extensão do punho, desvio radial/ulnar e flexão/extensão dos dedos. As informações coletadas são transmitidas e recebidas por meio de sistemas de comunicação sem fio, como Bluetooth. O aplicativo de software manipula mãos virtuais ou objetos virtuais em jogos de treinamento de acordo com os dados recebidos. Além disso, este sistema pode avaliar a amplitude de movimento ativo e passivo para cada movimento funcional (Shin et al., 2016).

Alguns dispositivos são do tipo sistemas vestíveis como, por exemplo, o Zishi que podem detectar movimentos compensatórios do tronco e do complexo do ombro e fornecem feedback em tempo real para dar suporte ao treino de reabilitação. O Zishi é formado por quatro partes: 1) uma central flexível equipada com 2 sensores; 2) unidade de sensor embutida em uma faixa de velcro elástica; 3) um tecido inteligente encaixado numa peça de roupa e inserida em pontos de conexão; 4) um aplicativo executado em um dispositivo portátil Android que suporta calibração para se adequar ao corpo do paciente e às necessidades do treinamento em específico, o qual fornece feedback de desempenho em tempo real e proporciona conhecimento resultado alcançado com o treinamento. Um ensaio clínico envolvendo pacientes com AVC demonstrou que eles são estimulados a usar o Zishi durante a reabilitação e consideram o sistema útil; alguns, porém, hesitam em usá-lo de forma independente quando comparado ao acompanhamento de fisioterapeutas, de forma que os autores alegam baixa adesão ao Zishi por parte dos pacientes que preferem a realização das tarefas de reabilitação num espaço clínico, incluindo a presença do profissional fisioterapeuta (Wang et al., 2018). 
Em relação aos produtos de TA que apoiam a realização de atividades com as mãos, Rodrigues, Muniz \& Xavier (2015) apresentaram uma adaptação funcional desenvolvida com Etil Vinil Acetato (EVA) para melhorar o desempenho de hansenianos com mão em garra durante a alimentação. O enfoque está em demonstrar a autopercepção dos pacientes acerca da melhora do seu desempenho no ato de se alimentar. A amostra do estudo foi composta por 20 pacientes que foram escolhidos de maneira randômica por sorteio, com um total de 10 sessões e com duração de 45 minutos cada. $\mathrm{O}$ resultado deste estudo demonstrou aumento no grau de independência durante a realização das atividades de vida diária, mostrando significância estatística ao comparar os resultados obtidos antes e depois do uso das adaptações. No entanto, este estudo não apresenta um processo de desenvolvimento de produto, assim como não faz uso da tecnologia de impressão 3D.

A Bicicleta Inteligente (Smart Bike) foi desenvolvida objetivando a monitorização contínua das características mecânicas e elétricas de cada ciclo gerado, juntamente com a resposta física e neuromuscular do ciclista durante a operação. Os dados capturados podem ser analisados para determinar quais características temporais exclusivas dos dados amostrados estão correlacionadas com o nível de habilidade motora do piloto. A correlação estabelecida permite a avaliação em tempo real do desempenho do ciclista durante uma sessão de ciclismo. Este design inovador incorpora drives e controles de alto desempenho e um servomotor de baixa inércia e alta potência para formar uma plataforma flexível e adaptável para apoiar estudos de pesquisa clínica de exercícios para pessoas com doença de Parkinson (Mohammadi-Abdar et al., 2015).

De acordo com Ireno et al., (2019), existem diferentes recursos, métodos e abordagens de intervenção terapêutica que buscam minimizar as dificuldades e facilitar a funcionalidade e a participação de crianças com PC em atividades cotidianas. Dentre eles, citam-se os recursos da TA como adjuvantes no tratamento de reabilitação, tais como as órteses. Essas possuem um papel fundamental, pois têm a função de manter e/ou promover a amplitude de movimento articular, a fim de substituir ou aumentar a função, prevenir ou corrigir deformidades, oferecer repouso articular e reduzir a dor. Esse autor aponta ainda para diversos benefícios advindos do uso de diferentes tipos de órteses em crianças com PC, como possibilidade para otimização do padrão de locomoção, redução da flexão plantar, melhora nos parâmetros qualitativos da marcha e no desempenho motor, bem como, redução no gasto energético.

Em um estudo realizado por Tavares et al., (2020), cujo objetivo principal foi identificar a importância de dispositivos tecnológicos na paralisia cerebral, os aparatos tecnológicos de interação citados na pesquisa para uso proveitoso na doença foram vídeo game Wii, projetores, tablets, mesas touchscreen, vocalizadores, mesas digitais e interativas, esteira suspensa, andadores e exoesqueletos. A associação das terapias com os aparatos tecnológicos e aparelhos para entretenimento tornou-se essencial no desenvolvimento de uma criança com PC.

\subsection{Dispositivos Inteligentes para a Marcha}

Com o advento da tecnologia, a implementação de soluções inteligentes no processo de reabilitação das mais diversas afecções neurológicas, permite o empoderamento do paciente e de seu respectivo cuidador. Além disso, essas ferramentas também possuem o potencial de reduzir a carga para os profissionais de saúde e apoiar o desenvolvimento de novas intervenções (Davies et al., 2016). Nesse sentido, diversos dispositivos podem ser utilizados para promover a facilitação e aprimoramento da marcha em indivíduos com sequelas de lesão cerebral.

\subsubsection{CPWalker}

O CPWalker consiste em uma plataforma robótica de reabilitação da marcha que é constituída por uma espécie de andador e um exoesqueleto. O CPWalker é utilizado em pessoas com PC, visando fornecer a essa população uma estrutura que irá reabilitar sua marcha para padrões fisiológicos, evitando a deformação óssea por desuso, como também, reduzindo o período de reabilitação após cirurgias ortopédicas multiníveis (Bayón et al., 2016; Bayón et al., 2016). Os sistemas incluídos, 
são: sistema de acionamento para fornecer o movimento de translação necessário para um treino de marcha em ambientes reais de reabilitação; sistema de suporte parcial de peso corporal (parcial body-weight support training, PBWS, sigla em inglês); sistema de controle da altura do quadril, afim de adaptar a plataforma às diferentes medidas antropométricas e um sistema exoesqueleto ajustável em seis graus de liberdade (6-DOF) para fornecer movimentos guiados no plano sagital. A faixa de velocidade do dispositivo é entre $[-0,561,+0,561] \mathrm{m} / \mathrm{s}$ (Bayón et al., 2016). Em estudo desenvolvido por Bayón e colaboradores (2016), foi comprovado que parâmetros espaço-temporais da marcha como velocidade média, cadência e comprimento do passo foram melhorados após utilização do CPWalker.

\subsubsection{Walkaide}

O WalkAide (Innovative Neurotronics Inc, Austin, Texas), é um sistema que oferece estimulação elétrica de superfície bifásica assimétrica para o nervo fibular comum, de forma que é acionado por um sensor de inclinação programado individualmente para facilitar a movimentação do pé durante a fase de balanço da marcha. Com o objetivo de aprimorar o padrão de marcha em indivíduos com lesões cerebrais, esse dispositivo apresenta algumas vantagens em relação a estimulação elétrica funcional (FES) convencional, por ser uma pequena unidade leve à bateria, e não apresentar nenhum efeito adverso (Elsner et al., 2021).

O sistema do WalkAide é composto por dois eletrodos dispostos e posicionados, um, posterior à cabeça da fíbula; e, o eletrodo "passivo" colocado sobre o ventre do músculo tibial anterior proximal, a fim de promover a dorsiflexão do tornozelo e completar o circuito elétrico (Damiano et al., 2013).

\subsubsection{Dispositivo Inteligente de Mudanças Rítmicas}

Um aplicativo de estimulação auditiva rítmica (RAS, do inglês: Rhythmic Auditory Stimulation) para ser utilizado em smartphones com sistema Android, foi desenvolvido com objetivo de melhorar a cadência e o desempenho da marcha em indivíduos com AVC. Este sistema consiste em três módulos: módulo de detecção do sinal de marcha, módulo de análise de marcha e módulo de alto-falante. O módulo de detecção do sinal de marcha recebe dados de movimentação da perna por meio de acelerômetros 3D (ShimmerTM) que são colocados acima do tornozelo esquerdo e direito. O módulo de análise da marcha analisa os dados de características de marcha dos acelerômetros 3D e gera RAS. O módulo de alto-falante fornece o RAS ao usuário. O aplicativo RAS possui sete fontes sonoras (CEG, CFA, ADG, palmas, clique, arma e robô) que podem ser selecionadas e aplicadas de acordo com a preferência do usuário (Hwi-Young et al., 2016; Hwi-Young et al., 2015).

\subsubsection{AGoRA Smart Walker}

O AGoRA Smart Walker é um andador robótico inteligente, montado em um robô comercial (Pioneer LX, Omron Adept, Amherst, NH, EUA). Este dispositivo tem como finalidade fornecer assistência à marcha em diferentes condições neurológicas por ter a capacidade de responder às intenções do usuário para locomover-se (Sierra et al., 2021; Sierra et al., 2019).

O AGoRA Smart Walker funciona a partir de diversos sensores e unidades de processamento que são utilizados em uma plataforma. Duas rodas motorizadas e duas rodas giratórias para propulsão e estabilidade; dois codificadores e uma unidade de medição inercial (IMU) para medir a posição, orientação e velocidade; deteç̧ão de luz 2D e sensor de alcance (LiDAR) (S300 Expert, SICK, Waldkirch, Alemanha) para detecção de ambiente; duas placas ultrassônicas para detecção do usuário e detecção de obstáculos de baixo nível; dois sensores de força tri-axial (MTA400, FUTEK, Irvine, CA, EUA) para estimar os comandos de navegação do usuário; uma câmera HD (LifeCam Studio, Microsoft, Redmond, WA, EUA) para 
detectar a presença das pessoas no ambiente e um localizador de alcance a laser 2D (LRF) (Hokuyo URG-04LX-UG01, Osaka, Japão) para a estimativa de marcha do usuário fazem parte do andador robótico (Sierra et al., 2021; Sierra et al., 2019).

\subsubsection{Realidade Virtual}

A realidade virtual (RV) pode ser inserida no tratamento de pacientes com lesões neurológicas por meio da utilização de dispositivos que promovam a interação do indivíduo com a simulação do ambiente. Para isso, existem equipamentos disponíveis comercialmente, como o Wii, óculos de RV ou até mesmo um protótipo desenvolvido por pesquisadores (Yang et al., 2016).

Yang e colaboradores (2016), demonstraram em seu estudo que a RV se mostrou como um recurso capaz de fornecer feedbacks sensoriais ricos por meio de pistas visuais e/ou cognitivas a fim de facilitar a aprendizagem, retenção e transferência motora em pacientes com DP. Essa população apresenta benefícios na execução de atividades com pistas externas, enquanto demonstram prejuízos nas tarefas com pistas internas Hshieh (2016, as cited in Yang et al., 2016).

\subsubsection{Cadeira de Rodas Inteligente}

Com o advento da tecnologia, atualmente, existem diferentes interfaces com vistas a facilitarem o deslocamento das pessoas que utilizam cadeira de rodas (CR). Dentre os modelos disponíveis, destacam-se o sistema de controle por joystick inteligente, que funciona por meio de gestos manuais visuais, sendo uma opção "mãos-livres" que não requer sensores fixados no corpo do indivíduo ou câmera especial na CR. O deslocamento da cadeira de rodas é depende de informações fornecidas a partir de gestos da mão do usuário, o qual indica a direção do movimento da $\mathrm{CR}$, e determina o percurso e destino final desejado com seleção da aceleração adequada. Essa interface é baseada principalmente em visão computacional, processamento de imagens e manipulação de pixels, para os quais existe uma biblioteca de código aberto chamada OpenCV (Open Source Computer Vision Library), consistindo em mais de 2500 algoritmos otimizados. Tal dispositivo pode ser direcionado especialmente a pessoas com deficiências graves nas extremidades superiores (Rabbi, 2018).

\subsubsection{Lokomat}

O Lokomat (Hocoma; Zurique, Suíça), refere-se a um exoesqueleto robótico que proporciona a execução da marcha em esteira em indivíduos com lesões neurológicas que comprometem a função dos membros inferiores, especialmente nas lesões medulares (Nam et al., 2017).

O exoesqueleto do Lokomat é acionado por unidades lineares para fornecer orientação para os membros inferiores, e segue um padrão de referência baseado na cinemática da marcha de pessoas saudáveis. O padrão de referência pode ser ajustado modificando os ângulos do quadril e do joelho para atender as necessidades de cada indivíduo (Kammen et al., 2020; Nam et al., 2017).

Tal equipamento é composto por um sistema de suporte de peso corporal (BWS), combinado com braços robóticos presos às pernas do usuário e uma esteira. Esse dispositivo pode ser ajustado às características antropométricas de cada indivíduo e tem sido utilizado em diferentes faixas etárias.

\subsection{Dispositivos Vestíveis}

Os dispositivos vestíveis, segundo Atshzar et al. (2020), são considerados como tecnologia utilizada para monitoração dos sinais vitais e mostram informações aos usuários através de biofeedback tátil, vibratório ou por eletroestimulação para melhorar, assistir e aumentar as capacidades de realização dos movimentos. 
Dispositivos vestíveis desenvolvidos na atualidade são desenhados para todas as partes do corpo humano e são classificados em 3 categorias: equipamentos para a cabeça, membros e tronco (Lu et al., 2020).

Os dispositivos vestíveis surgiram em decorrência desta miniaturização, que possibilita um desempenho maior em computadores pessoais e consistem em micro-sensores dispostos em relógios, pulseiras, óculos, tornozeleiras, tênis, etc (Remédio, 2019). Esses sensores vestíveis têm o potencial de fornecer um diagnóstico precoce aos pacientes que estão numa faixa etária avançada ou em condições desfavoráveis de saúde e servem para monitorar sintomas leves de alteração de frequência cardíaca, pressão arterial, saturação de oxigênio, temperatura e mobilidade em pessoas que não precisam da hospitalização. Com o uso desse artificio inteligente, os dados coletados podem ser processados na nuvem e qualquer anormalidade pode ser detectada usando modelos computacionais (Atshzar et al., 2020).

A maioria dos dispositivos vestíveis para membros superiores são relógios inteligentes, braceletes e outros acessórios que podem ser usados para monitorar parâmetros fisiológicos como temperatura, frequência cardíaca, níveis de exposição ultravioleta e atividades da vida diária. Segundo Remédio (2019) a partir do uso de tais sensores, pode-se extrair diversas informações corporais em tempo real, e realizar análises sobre as informações anteriormente citadas com uma abordagem pessoal e única. Em contraste com a terapia convencional, na qual o paciente faz o treinamento passivo diário repetitivo, um sistema robótico ativo-assistido desenvolvido para reabilitação neurológica pode capturar sinais biopotenciais dos pacientes, decodificar os seus desejos de movimento e ativar respostas ativas para suas intenções. Já foi provado que tais sistemas robóticos são mais eficazes que os métodos passivos e podem melhorar os resultados terapêuticos (Yang et al., 2018).

Os sistemas baseados em sensores vestíveis extraem e analisam também dados cinemáticos, e se destacam por auxiliar no diagnóstico, na reabilitação, no acompanhamento pós tratamento, assim como, no tratamento das lesões musculoesqueléticas e neurológicas (Carnevale et al., 2019). Em revisão sistemática envolvendo 73 artigos sobre uso desses dispositivos vestíveis de membros superiores, os autores concluíram que essa tecnologia tem potencial para fornecer informações clínicas quantitativas e importantes sobre a qualidade de movimento, e, por direcionar os ganhos obtidos no processo de reabilitação (Carnevale et al., 2019).

Existem também dispositivos robóticos utilizados na recuperação de membros superiores, que promovem a terapia por meio de sustentação do membro, facilitando a realização de movimentos ativos de ombro, cotovelo, punho e mão, os quais, podem ser associados a jogos de RV. Assim, o equipamento monitora o desempenho em tarefas funcionais, reconhece as dificuldades e propõe desafios em tempo real e personalizados para cada tipo de tratamento (Palermo et al., 2018).

A maioria dos dispositivos dos membros inferiores aparecem na forma de sapatos e meias que monitoram parâmetros relacionados ao movimento e são principalmente usados no campo da reabilitação (Lu et al., 2020). Quando se consideram as pistas externas, por exemplo, dispositivos ambulatoriais foram desenvolvidos para permitir que pistas visuais sejam projetadas ao longo do caminho a ser percorrido pelo paciente em contraste com as pistas visuais tradicionais que eram limitadas a determinados locais do espaço doméstico ou no trajeto específico da marcha. Um exemplo de pista visual é o sapato a laser que é equipado com uma ponteira a laser, a qual projeta linhas transversais no campo visual do paciente. Essas linhas transversais podem ser especificamente úteis para minimizar o congelamento da marcha em pacientes com a doença de Parkinson, embora evidências atuais sejam limitadas a um único relato de caso (Ginis et al., 2018).

Estudo semelhante foi realizado por Argañarás et al., (2021) envolvendo dispositivo vestível do tipo sapato com um sistema de radar embutido para detecção de obstáculos e degraus de escadas, objetivando a prevenção de quedas. Nele, um protótipo de sapato com radar foi desenhado, construído e experimentalmente testado, e os resultados mostraram-se promissores na análise da marcha e na prevenção de quedas a partir dessa tecnologia.

Os dispositivos vestíveis do dorso incluem trajes, cintos e roupa íntima. Nos últimos anos, o desenvolvimento rápido da tecnologia de materiais e tecnologia de detecção material facilitou a manufatura de produtos eletrônicos embutidos em 
tecidos que podem ser usados em várias aplicações biomédicas. Em 2009, um sistema de vestuário que dá acesso à Internet foi desenvolvido pelo Laboratório de Tecnologia de Mídia do Instituto de Tecnologia de Massachusetts e marcou a chegada de uma nova era de têxteis eletrônicos (Lu et al., 2020). Um ensaio clínico randomizado realizado em 2017 na Nova Zelândia envolvendo trabalhadores que sofriam de lombalgia avaliou a eficácia de um dispositivo do tamanho de um pager capaz de detectar variações nos movimentos do tronco e sinalizar aos pacientes os padrões anormais da postura que prejudicam a coluna vertebral com vistas a prevenir os malefícios da dor na coluna vertebral (Ribeiro et al., 2017).

Dispositivos vestíveis parecem muito úteis para desordens do equilíbrio e ajudam pessoas com Doença de Parkinson a reduzir o balanço do tronco após um programa de exercícios supervisionados Rossi-Izquierdo (2018 as cited in Bao, 2018). Ensaio clínico randomizado mostrou a eficácia de um dispositivo do tipo cinto elástico criado para possibilitar a realização de exercícios de equilíbrio caseiros. Por meio de estímulos vibratórios e de uma interface didática, o grupo experimental mostrou melhora do equilíbrio, da confiança visual e vestibular e nas respostas de alguns testes clínicos de equilíbrio quando comparado ao grupo controle (Bao, 2018).

\subsection{Módulos de Interação de Interface e Feedback sobre Exercício Usados em Home Rehabilitation (Reabilitação em} Casa)

Uma vez que a tecnologia, na situação em questão, tem por intuito favorecer o processo de reabilitação de indivíduos que apresentam sequela de acometidos por algumas situações como, por exemplo, o AVC, por exemplo, faz-se necessário o desenvolvimento de ferramentas que possam fornecer o processo de reabilitação continuada, durante o processo de reabilitação em domicílio. O uso da internet das coisas e Tecnologia da Informação e Comunicação (TIC) podem ser úteis durante o processo de reinserção social e no trabalho dessas pessoas com sequelas sensório motoras (Cogollor, 2018).

Por meio do uso dos equipamentos eletrônicos integrados ao ambiente domiciliar, é possível desenvolver atividades das mais diversas maneiras, como por exemplo o uso da reabilitação à distância, em que o paciente faz uso de hardwares como computadores ou smartphones de forma a proporcionar comunicação entre paciente e terapeuta. Essa comunicação, não se dá apenas por meio de chamada de vídeo, mas também por feedbacks gerados por sensores instalados nas vestimentas dos pacientes, por exemplo (Bisio et al., 2019).

Portanto, o fato do paciente enviar feedback instantâneo por meio da internet para o terapeuta, favorece algumas situações como a diminuição de custo e tempo no deslocamento em detrimento da atividade presencial. A partir do uso da reabilitação à distância possibilita-se a prescrição do exercício físico, de extrema importância para entender o comportamento do corpo desses indivíduos durante a prática da atividade domiciliar de reabilitação. A inclusão da tecnologia, por meio de smartphone e tablets, promoveu otimização de acesso a mais pessoas com sequelas que dependem da prescrição e acompanhamento da reabilitação domiciliar (Emmerson et al., 2018).

Em estudo realizado por Emmerson et al. (2018), foi demonstrado achados favoráveis a este novo modelo de interface de condução de programas de exercício físico para pessoas idosas em processo de reabilitação pós AVC. No estudo, foi possível obter relatos de alguns participantes que demonstraram aumento da força e/ou da amplitude de movimento dos membros superiores. Com relação ao ganho funcional, foi identificado um grande leque de benefícios, dentre eles, a motivação para complementar os exercícios domiciliares, promovendo a continuidade das instruções e, melhorando a organização do desenvolvimento dessas atividades em casa, com consequente adesão ao programa sugerido.

Portanto, é possível observar que a inclusão de sensores nas vestes, favorece o acompanhamento dos pacientes por parte dos profissionais fisioterapeutas ao longo do processo de reabilitação, facilitando o trabalho dos terapeutas e dos indivíduos envolvidos nesse processo, uma vez que eles podem ter acesso às informações nas "palmas das mãos", com o uso 
de smartphone, tablets e computadores portáteis. Além disso, a comunicação paciente-terapeuta pode se dar de maneira mais efetiva e eficaz, à distância e instantaneamente.

\subsection{Smart Rehabilitation e Fisioterapia}

Como a implementação de TA vem aumentando, junto com a variedade de dispositivos disponíveis, é de extrema importância que a equipe de reabilitação esteja ciente dos aparelhos disponíveis, bem como do impacto do mesmo no processo de reabilitação. É de extrema utilidade que fisioterapeutas experimentem e enviem sugestões quanto ao design, possíveis melhorias e ajustes a serem feitos nesses equipamentos, baseados em sua experiência clínica (Lobo et al., 2019).

A utilização de robôs na reabilitação humana é um exemplo da influência benéfica que a tecnologia concede nos tratamentos, visto que ela dinamiza e proporciona inúmeros resultados positivos no processo de evolução e aquisição de ganhos na fisioterapia. Outrossim, percebe-se que os mecanismos da robótica proporcionam a execução de determinados exercícios de modo controlado, eficaz e confiável, sendo validado na literatura como fator essencial para a melhoria da habilidade motora (Peres et al., 2018).

Além dos aspectos anteriormente citados, o lado lúdico dos robôs possibilita aos pacientes maior interação, envolvimento e diversão durante a terapia, o que viabiliza a permanência desses pacientes no tratamento. Nessa perspectiva, essa tecnologia dispõe de requisitos importantes e procurados, frequentemente, por fisioterapeutas e médicos (Cogliatti, 2019).

Em um estudo realizado por Azevedo e Maltempi (2021), foi observado que os atendimentos fisioterapêuticos juntamente com os jogos e dispositivos podem promover um aumento na liberação de dopamina na DP, tendo em vista que se origina pelo processo de aprendizagem e das características ambientais. Os jogos e dispositivos eletrônicos incentivam o paciente a um maior nível de envolvimento e identificação com os objetivos e metas das sessões de fisioterapia, possibilitando maior motivação durante o tratamento.

Os artigos científicos pesquisados nesse estudo apontam a participação crucial do fisioterapeuta no processo de reabilitação dos pacientes que usam tecnologias smarts. Em todos os estudos revisados nota-se a participação desse profissional na educação do uso dos dispositivos de tecnologia smart e no acompanhamento dos participantes, no uso adequado dos instrumentos vestíveis, na boa utilização das tecnologias de interface por vídeo touch screen e na prescrição dos programas de exercícios de reabilitação (Emmerson et al., 2018; Wang et al., 2018; Bayón et al., 2016).

\subsection{Limitações desta Revisão}

O desenvolvimento tecnológico ainda está sendo processado e incorporado de forma mais solidificada aos processos de reabilitação, por isso ainda existem diversas lacunas a serem exploradas e preenchidas.

Dentre as principais limitações que podem ser citadas, podemos observar limitações de estudos por idioma abordado, visto que algumas revisões se encontravam disponíveis em inglês, espanhol e alguns estudos em português. Gerando, assim, uma limitação da obtenção das informações já coletadas por outros pesquisadores (Kettlewell \& Jade, 2019). Em seu trabalho, Kettlewell, e Jade (2019), observaram que outra limitação pode ser o fato de os desfechos serem definidos a partir do equipamento utilizado, e, não por meio do objetivo a ser alcançado, gerando, assim, uma ampla produção voltada para a especificidade do equipamento e não do objetivo em comum.

Além das limitações metodológicas, é possível identificar também aquelas que são voltadas para a inserção do uso dessas tecnologias na prática diária da fisioterapia. Em função do alto custo, a TA, limita a quantidade de usuários que podem ter acesso ao serviço (Lobo et al., 2019). Com baixo quantitativo de usuários das tecnologias, limitam-se as possibilidades de investigação a respeito das interações dos indivíduos e as TA. 
Não somente as condições financeiras podem limitar o acesso, mas também o fato de que a proporção de pessoas usuárias dos computadores diminui com a idade (Almeida et al., 2012). Portanto, dado que 69\% das pessoas que têm o AVC estão na faixa etária acima dos 65 anos (Australian Bureau of Statistics, 2016), uma consideração é se a idade seria uma barreira à incorporação da tecnologia smart no processo de reabilitação e reinserção social do adulto com sequela neurológica.

Relatos de estudos com foco no grupo de idosos consideraram o uso da tecnologia na reabilitação como um problema, já que os próprios participantes dos estudos atribuem à sua idade, dificuldades em se beneficiar dessas tecnologias, devido à pouca familiaridade em lidar com os dispositivos smarts (Emmerson et al., 2018).

Em decorrência de equivalente observação, um ensaio clínico randomizado, recente, comparou o uso da tecnologia dos Tablets no uso de programas de exercício caseiros em pacientes pós AVC com programas de exercício físico prescritos em papel (Emmerson et al., 2018). Foi possível observar que a aceitabilidade da tecnologia no processo de recuperação de idosos pós AVC não houve diferença entre o grupo usando tablet e o grupo usando as informações em papel (Emmerson et al., 2018), fato que sugere a possibilidade de uma adesão desse público, apesar da dificuldade que eles relatam ter para o uso da tecnologia.

A partir das limitações observadas nos estudos citados, faz-se necessário uma melhor delimitação metodológica para abarcar situações mais generalistas e que direcionem o desfecho para os resultados esperados, e não para a avaliação específica dos equipamentos. Outrossim, as TA ainda são de difícil acesso para alguns públicos em face dos custos financeiras relativos à aquisição dos dispositivos, por exemplo.

\section{Conclusão}

Com o desenvolvimento científico e tecnológico, espera-se que os dispositivos smarts possam vir a desempenhar um papel importantíssimo no campo da ciência e serem integrados à rotina das pessoas com disfunções neuromusculoesqueléticas. Apesar das limitações e disponibilidade dessa tecnologia à população socioeconomicamente desfavorecida, os dispositivos inteligentes são uma opção promissora e futuros aliados dos profissionais da saúde nos pequenos e grandes centros de reabilitação física do Brasil e do mundo. Apoiados pela literatura recente, os riscos e benefícios da Smart Rehabilitation e, o saldo do uso da SR parece ser bastante positivo e, ao que tudo indica, a ciência avança no sentido da "nova era" da medicina e áreas afins, quando se pensa na autonomia, na independência, na recuperação motora e suas repercussões na integralidade da saúde humana.

Não se pode, por outro lado negar, a falta de uma ampla discussão sobre o assunto entre as mais diversas áreas da ciência, envolvendo a engenharia, todas as categorias da saúde, usuários, pesquisadores e formuladores de políticas públicas, porque há algumas lacunas sem respostas quando se analisa a viabilidade, confiabilidade e, principalmente, no quanto essa tecnologia é conhecida no contexto clínico e hospitalar.

Cabe aos futuros estudos, uma discussão sobre a implementação e acessibilidade dessas ferramentas na tão amplificada e estratificada sociedade brasileira. Atenção deve ser dada aos ensaios clínicos randomizados com amostras representativas e com metodologia rica o suficiente para responder, por exemplo, o quão eficaz seria a inserção dessas tecnologias no contexto do Sistema Único de Saúde. Por último, tornar a SR socialmente popular para fomentar a substituição de tecnologias obsoletas pelas novas ferramentas promissoras.

\section{Agradecimentos}

Os autores deste artigo agradecem fortemente à doutora, mestre e professora Adriana Carla Costa Ribeiro Clementino por acreditar em sua proposta na produção científica e por colaborar no desenvolvimento deste artigo. 


\section{Referências}

Albuquerque, V. S., Fernandes, L. P., \& Mármora, C. H. C. (2019). O uso de dispositivos auxiliares para marcha em idosos e sua relação com autoeficácia para quedas. Revista Hospital Universitário Pedro Ernesto, 17(2), 51-56. https://doi.org/10.12957/rhupe.2018.40858.

Almeida, O. P., Yeap, B. B., Alfonso, H., Hankey, G. J., Flicker, L., \& Norman, P. E. (2012). Older Men Who Use Computers Have Lower Risk of Dementia. PLOS ONE, 7(8), 1-6. https://doi.org/10.1371/journal.pone.0044239.

Argañarás, J. G., Wong, Y. T., Begg, R., \& Karmakar, N. C. (2021). State-of-the-art wearable sensors and possibilities for radar in fall prevention. Sensors, 21(20), 1-23. https://doi.org/10.3390/s21206836.

Atashzar, S. F., Carriere, J., \& Tavakoli, M. (2021). Review : How Can Intelligent Robots and Smart Mechatronic Modules Facilitate Remote Assessment, Assistance, and Rehabilitation for Isolated Adults With Neuro-Musculoskeletal Conditions?. Frontiers and Robotics and AI, 8, 119. https://doi.org/10.3389/frobt.2021.610529.

Azevedo, G. T., \& Maltempi, M. V. (2021). Invenções robóticas para o Tratamento de Parkinson: pensamento computacional e formação matemática. Bolema: Boletim de Educação Matemática, 35(69), 63-88. https://doi.org/10.1590/1980-4415V35N69A04.

Bao, T., Carender, W. J., Kinnaird, C., Barone, V. J., Peethambaran, G., Whitney, S. L., Kabeto, M., Seidler, R. D., \& Sienko, K. H. (2018). Effects of longterm balance training with vibrotactile sensory augmentation among community-dwelling healthy older adults: A randomized preliminary study. Journal of NeuroEngineering and Rehabilitation, 15(1), 1-13. https://doi.org/10.1186/s12984-017-0339-6.

Brasil (2009). Tecnologia Assistiva. Brasília

Barreto, G. F. M., Aversari, L. O. C., Barreto, R. G. (2019). Gerência de Projetos em Computação Vestível: Diretrizes para o Desenvolvimento de Produtos Vestíveis Inteligentes, 388-416. Atena editora.

Bayón, C., Lerma, S., Ramírez, O., Serrano, J. I., Del Castillo, M. D., Raya, R., Martínez, I., \& Rocon, E. (2016). Locomotor training through a novel robotic platform for gait rehabilitation in pediatric population: short report. Journal of NeuroEngineering and Rehabilitation, https://doi.org/10.1186/s12984-0160206-x.

Bayón, C., Ramirez, O., Del Castillo, M. D., Serrano, J. I., Raya, R., Belda-Lois, J. M., Poveda, R., Mollà, F., Martín, T., Martínez, I., Lara, S. L., \& Rocon, E. (2016). CPWalker: Robotic Platform for Gait Rehabilitation in Patients with Cerebral Palsy. In: 2016 IEEE International Conference on Robotics and Automation (ICRA), 3736-3741.

Bisio, I., Garibotto, C., Lavagetto, F., \& Sciarrone, A. (2019). When eHealth Meets IoT: A Smart Wireless System for Post-Stroke Home Rehabilitation. IEEE Wireless Communications, 26(6), 24-29.

Carnevale, A., Longo, U. G., Schena, E., Massaroni, C., Lo Presti, D., Berton, A., Candela, V., \& Denaro, V. (2019). Wearable systems for shoulder kinematics assessment: A systematic review. BMC Musculoskeletal Disorders, 20(1), 1-24. https://doi.org/10.1186/S12891-019-2930-4/FIGURES/3

Caro, C. C., Costa, J. D., \& Cezar da Cruz, D. M. (2018). O uso de dispositivos auxiliares para a mobilidade e a independência funcional em sujeitos com Acidente Vascular Cerebral. Cadernos Brasileiros de Terapia Ocupacional, 26(3), 558-568. https://doi.org/10.4322/2526-8910.CTOAO1117

Casarin, S. T., Porto, A. R., Gabatz, R. I. B., Bonow, C. A., Ribeiro, J. P., \& Mota, M. S. (2020). Tipos de revisão de literatura: considerações das editoras do Journal of Nursing and Health / Types of literature review: considerations of the editors of the Journal of Nursing and Health. Journal of Nursing and Health, 10(5), 1-7. https://doi.org/10.15210/jonah.v10i5.19924

Cogollor, J. M., Rojo-lacal, J., Hermsdörfer, J., Ferre, M., Waldmeyer, M. T. A., Giachritsis, C., Armstrong, A., Martinez, J. M. B., Loza, D. A. B, \& Sebastián, J. M. (2018). Evolution of Cognitive Rehabilitation After Stroke From Traditional Techniques to Smart and Personalized Home-Based Information and Communication Technology Systems : Literature Review. JMIR rehabilitation and assistive technologies, 5(1). https://doi.org/10.2196/rehab.8548.

Coimbra, T. M., Ezequiel, C. T., Moreira, D. S., Morita, M. P. A., Castiglioni, L., \& Bianchin, M. A. (2018). Comunicação Alternativa Ampliada na Esclerose Lateral Amiotrófica: a tecnologia a favor da reabilitação. Arquivos de Ciências da Saúde - Faculdade de Medicina de São José do Rio Preto - FAMERP, 25(3), 22. http://dx.doi.org/10.17696/2318-3691.25.3.2018.1054.

Damiano, D. L., Prosser, L. A., Curatalo, L. A., \& Alter, K. E. (2013). Muscle Plasticity and Ankle Control After Repetitive Use of a Functional Electrical Stímulation Device for Foot Drop in Cerebral Palsy. Neurorehabilitation Neural Repair, 27(3), 200-207. https://doi.org/10.1177/1545968312461716.

Davies, R. J., Parker, J., Mccullagh, P., \& Zheng, H. (2016). A Personalized Self-Management Rehabilitation System for Stroke Survivors : A Quantitative Gait Analysis Using a Smart Insole. JMIR Rehabil Assist Technol, 3(2), 1-13. https://doi.org/10.2196/rehab.5449.

Elsner, V. R., Trevizol, L., De Leon, I., Silva, M., Weiss, T., Braga, M., Pochmann, D., Blembeel, A. S., Dani, C., \& Boggio, E. (2021). Therapeutic effectiveness of a single exercise session combined with WalkAide functional electrical stimulation in post-stroke patients : a crossover design study. Neural Regen Res. 16(5), 805-812.

Emmerson, K. B., Harding, K. E., Lockwood, K. J., \& Taylor, N. F. (2018). Home exercise programs supported by video and automated reminders for patients with stroke: A qualitative analysis. Australian Occupational Therapy Journal, 65(3), 187-197. https://doi.org/10.1111/1440-1630.12461.

Finch, T. R., Finch, P. D., \& Cook, D. R. (2016). Assistive apparatus for hand held vessels or items. U.S. Patent, $237(9), 796$.

Ginis, P., Nackaerts, E., Nieuwboer, A., \& Heremans, E. (2018). ScienceDirect Cueing for people with Parkinson's disease with freezing of gait : A narrative review of the state-of-the-art and novel perspectives. Annals of Physical and Rehabilitation Medicine.61, 407-413.

Ireno, J. M., Chen, N., Zafani, M. D., \& Baleotti, L.R. (2019). O uso de órteses em crianças com paralisia cerebral. Cardenos Brasileiros de Terapia Ocupacional, 27(1), 35-44. https://doi.org/10.4322/2526-8910.ctoAO1612. 
Kettlewell, J., das Nair, R., \& Radford, K. (2019). A systematic review of personal smart technologies used to improve outcomes in adults with acquired brain injuries. Clinical Rehabilitation, 33(11), 1705-1712. https://doi.org/10.1177/0269215519865774.

Ko, B. W., Lee, H. Y., \& Song, W. K. (2016). Rhythmic auditory stimulation using a portable smart device: Short-term effects on gait in chronic hemiplegic stroke patients. Journal of Physical Therapy Science, 28(5), 1538-1543. https://doi.org/10.1589/jpts.28.1538

Korzeniewska, E., Krawczyk, A., Mróz, J., Wyszyńska, E., \& Zawiślak, R. (2020). Applications of smart textiles in post-stroke rehabilitation. Sensors (Switzerland), 20(8), 1-12. https://doi.org/10.3390/s20082370

Lee, H. Y., Ko, B. H., Song, W. K., Kim, H., \& Shin, J. H. ( 2015). Rhythmic Auditory Stimulation for Robot-Assisted Gait Rehabilitation: a preliminar study. IEEE International Conference on Advanced Intelligent Mechatronics (AIM). https://doi.org/10.1109/AIM.2015.7222569.

Llorente, J. O. (2019). The potential of error-related potentials. Analysis and decoding for control, neuro-rehabilitation and motor substitution. Fundácion Dialnet. Available in: https://dialnet.unirioja.es/servlet/tesis?codigo=257829. Access in: 19 november 2021.

Lobo, M. A., Hall, M. L., Greenspan, B., Rohloff, P., Prosser, L. A., \& Smith, B. A. (2019). Wearables for Pediatric Rehabilitation: How to Optimally Design and Use Products to Meet the Needs of Users. Physical Therapy, 99(6), 647-657. https://doi.org/10.1093/PTJ/PZZ024.

Lu, L., Zhang, J., Xie, Y., Gao, F., Xu, S., Wu, X., \& Ye, Z. (2020). Wearable health devices in health care: Narrative systematic review. JMIR MHealth and UHealth, 8(11). https://doi.org/10.2196/18907.

Mendes, G. C., Barros, F. S., \& Nohama, P. (2018). Tecnologia assistiva lúdica para ganho de funcionalidade. Revista da Universidade Vale do Rio Verde, 16(2), 1-10. http://dx.doi.org/10.5892/ruvrd.v16i2.4975.g10951282.

Mohammadi-Abdar, H., Ridgel, A. L., Discenzo, F. M., \& Loparo, K. A. (2016). Design and Development of a Smart Exercise Bike for Motor Rehabilitation in Individuals with Parkinson's Disease. IEEE/ASME Transactions on Mechatronics: A Joint Publication of the IEEE Industrial Electronics Society and the ASME Dynamic Systems and Control Division, 21(3), 1650-1658. https://doi.org/10.1109/TMECH.2015.2508030

Monge, J., \& Postolache, O. (2018). Augmented reality and smart sensors for physical rehabilitation. In: 2018 International Conference and Exposition on Electrical And Power Engineering (EPE). International Conference and Exposition on Electrical and Power Engineering (EPE), 1010-1014. https://doi.org/10.1109/ICEPE.2018.8559935.

Morgado, F. L., \& Rossil, L. A. (2011). Correlação entre a escala de coma de Glasgow e os achados de imagem de tomografia computadorizada em pacientes vítimas de traumatismo crânio encefálico. Radiologia Brasileira, 44(1), 35-41. https://doi.org/10.1590/S0100-39842011000100010.

Nam, K. Y., Kim, H. J., Kwon, B. S., Park, J., Lee, H. J., \& Yoo, A. (2017). Robot-assisted gait training (Lokomat) improves walking function and activity in people with spinal cord injury: a systematic review. Journal of NeuroEngineering and Rehabilitation, 14(1), 24. https://doi.org/10.1186/s12984-017-0232-3.

Neto, L. L., Júnior, M. C. F., \& Chun, R. Y. S. (2021). Amyotrophic lateral sclerosis, dysarthria, and language disorders - type of reserach and approaches in diferente areas: an integrative literature review. Revista CEFAC, 23(1), 01-13. http://dx.doi.org/10.1590/1982-0216/20212318220.

Neto, H. P., Grecco, Christovão, T. C. L., L. A. C., Braun, L. A., Giannasi, L. C., Salgado, A. S. I., Moura, R. C. F., Carvalho, P. T. C., Corrêa, J. C. F., Sampaio, L. M. M., Galli, M. \& Oliveira, C. S. (2012). Effect of posture-control insoles on function in children with cerebral palsy: Randomized controlled clinical trial. BMC Musculoskelet Disord, 13(193). https://doi.org/10.1186/1471-2474-13-193.

Palermo, E., Hayes, D. R., Russo, E. F., Calabrò, R. S., Pacilli, A., \& Filoni, S. (2018). Translational effects of robot-mediated therapy in subacute stroke patients: an experimental evaluation of upper limb motor recovery. PeerJ, 6(1), e5544. https://doi.org/0.7717/peerj.5544.

Peres, L. W., Leite, A. C. A. B., Alvarenga, W. de A., Ghazaoui, M. M. Al, Rahall, T. M., \& Nascimento, L. C. (2018). Estratégias lúdicas na reabilitação motora de crianças com paralisia cerebral: revisão integrativa. Revista Eletrônica de Enfermagem, 20. https://doi.org/10.5216/REE.V20.50936

Porto, J. M., Iosimuta, N. C. R., Coelho, A. C., \& Abreu, D. C. C. (2019). Recomendações para prescrição de dispositivos auxiliares da marcha em idosos. Revista Acta Fisiátrica, 26(3), 171-175. https://doi.org/10.11606/issn.2317-0190.v26i3a166646.

Postolache, O., Pereira, J. M. D., Viegas, V. \& Pedro, L. (2015). Smart Walker Solutions for Physical Rehabilitation. IEEE Instrumentation and Measurement Magazine, 18(5), 21-30. https://doi.org/10.1109/MIM.2015.7271223.

Rabbi, Y., Mrabet, M., \& Fnaiech, F. (2018). Intelligent Control Wheelchair Using a New Visual Joystick. Journal of Healthcare Engineering, $2018,6083565$. https://doi.org/10.1155/2018/6083565.

Remédio, T. P. (2019). Reabilitação fisioterapêutica por meio de jogos digitais: uma abordagem baseada em lógica fuzzy, camera de profundidade e dispositivos vestíveis. Dissertação (Mestrado em Ciência da Computação) - Instituto de Biociências, Letras e Ciências Exatas, Universidade Estadual Paulista "Júlio de Mesquita Filho", 1-77.

Ribeiro, D. C., Milosavljevic, S., Terry J., \& Abbott, J. H. (2017). Effectiveness of a lumbopelvic monitor and feedback device to change postural behaviour: a protocol for the ELF cluster randomised controlled trial. BMJ Open, 7(1). http://dx.doi.org/10.1136/bmjopen-2016-015568.

Rodrigues Junior, J. L., Muniz, L. S., \& Xavier, M. B. (2015). A utilização da tecnologia assistiva para alimentação na melhora do desempenho ocupacional de hansenianos com mão em garra. Hansen International, 39(1), 22-29.

Shin, J. H., Kim, M. I., Lee, J. Y., Jeon, Y. J., Kim, S., Lee, S., Seo, B., \& Choi, Y. (2016). Effects of virtual reality-based rehabilitation on distal upper extremity function and health-related quality of life: a single-blinded, randomized controlled trial. Journal of NeuroEngineering and Rehabilitation, 13 , 17. https://doi.org/10.1186/s12984-016-0125-x.

Sierra M., S. D., Garzón, M., Múnera, M., \& Cifuentes, C. A. (2019). Human-Robot-Environment Interaction Interface for Smart Walker Assisted Gait: AGoRA Walker. Sensors 2019, 19(13), 2897. https://doi.org/10.3390/S19132897. 
Research, Society and Development, v. 11, n. 1, e34111124856, 2022

(CC BY 4.0) | ISSN 2525-3409 | DOI: http://dx.doi.org/10.33448/rsd-v11i1.24856

Sierra, S. D., Múnera, M., Provot, T., Bourgain, M., \& Cifuentes, C. A. (2021). Evaluation of Physical Interaction during Walker-Assisted Gait with the AGoRA Walker: Strategies Based on Virtual Mechanical Stiffness. Sensors, 21(9), 3242. https://doi.org/10.3390/s21093242.

Silva, P. M. M., Ledycnarf, J. H., \& Morya, E. (2017). Inovação tecnológica na formação do fisioterapeuta. Revista Cadernos de Educação, Saúde e Fisioterapia, 4(8).

Tavares, C. S. M., Machado, B. R., Bischoff, B. M., \& Scoz, M. (2020). Possibilidades da Tecnologia Touchscreen para desenvolvimento motor e inclusão digital de pessoas com Paralisia Cerebral. Centro de Estudios en Diseño y Comunicación, 83, 176-187. http://dx.doi.org/10.18682/cdc.vi83.3738.

Kammen, K. K., Reinders-Messelink, H. A., Elsinghorst, A. L., Wesselink, C. F., Vries, B. M., Woude, L. H. V., Boonstra, A. M., \& Otter, R. (2020) Amplitude and stride-to-stride variability of muscle activity during Lokomat guided walking and treadmill walking in children with cerebral palsy. European Journal of Paediatric Neurology, 29, 108-117. https://doi.org/10.1016/j.ejpn. 2020.08.003.

Wang, Q., Timmermans, A., Chen, W., Jia, J., Ding, L., Xiong, L., Rong, J., \& Markopoulos, P. (2018). Stroke Patient's Acceptance of a Smart Garment for Supporting Upper Extremity Rehabilitation. IEEE Journal of Translational Engineering in Health and Medicine, 6, 2101009. https://doi.org/10.1109/JTEHM.2018.2853549.

Wolf, S. L., Winstein, C. J., Miller, J. P., Taub, E., Uswatte, G., Morris, D., Giuliani, C., Light, K. E., \& Nichols-Larsen, D. (2006). Effect of constraintinduced movement therapy on upper extremity function 3 to 9 months after stroke: the EXCITE randomized clinical trial. Jama, 296(17), 2095-2104. https://doi.org/ 10.1001/jama.296.17.2095.

Yang, G., Deng, J., Pang, G., Zhang, H., Li, J., Deng, B., Pang, Z., Xu, J., Jiang, M., Liljeberg, P., Xie, H., \& Yang, H. (2018). An IoT-Enabled Stroke Rehabilitation System Based on Smart Wearable Armband and Machine Learning. IEEE Journal of Translational Engineering in Health and Medicine, 6. https://doi.org/10.1109/JTEHM.2018.2822681.

Yang, W. C., Wang, H. K., Wu, R. M., Lo, C. S., \& Lin, K. H. (2016). Home-based virtual reality balance training and conventional balance training in Parkinson's disease: A randomized controlled trial. Journal of the Formosan Medical Association = Taiwan Yi Zhi, 115(9), 734743. https://doi.org/10.1016/J.JFMA.2015.07.012. 